\title{
Benefits of intravascular laser Irradiation of Blood on Motor and Sensory Recovery Viewing from Brain Function Images: Portrait of a Case with Chronic Sjögren's Syndrome, Transverse Myelitis, and Guillain- Barré Syndrome
}

\author{
Esther Yi Liu ${ }^{1}$ and Shin-Tsu Chang*2,3 \\ ${ }^{1}$ Department of Medical Education, Taichung Veterans General Hospital, Taiwan \\ ${ }^{2}$ Department of Rehabilitation, Taichung Veterans General Hospital, Taiwan
}

${ }^{3}$ Department of Physical Medicine and Rehabilitation, Tri-Service General Hospital, School of Medicine, National Defense Medical Center, Taipei, Taiwan

*Corresponding author: Shin Tsu Chang, Department of Physical Medicine and Rehabilitation, Tri-Service General Hospital, School of medicine, National Defense Medical Center, Neihu District, Taipei, Taiwan

\section{ARTICLE INFO}

Received: 彗 February 04, 2019

Published: 蔧 February 14, 2019

Citation: Esther Yi Liu, Shin-Tsu Chang. Benefits of intravascular laser Irradiation of Blood on Motor and Sensory Recovery Viewing from Brain Function Images: Portrait of a Case with Chronic Sjögren's Syndrome, Transverse Myelitis, and Guillain-Barré Syndrome. Biomed J Sci \& Tech Res 14(4)-2019. BJSTR. MS.ID.002572.

\author{
ABSTRACT
}

Transverse myelitis is an autoimmune disease and often causes paralysis of the lower limbs. The Guillain-Barré syndrome is characterized as sensorimotor disturbance and progressive limbs weakness from lower limbs and progresses symmetrically upward. Deafferent pain syndrome is also common. Combination of both diseases will worsen the consequences, and results in various disabilities. Conventional treatments cannot achieve the fast recovery of the motor and sensory dysfunction, and the intravascular laser irradiation of blood comes with the tide of fashion, which has been reported with efficacy in facilitating the circulation and increased wound healing rate. We herein report a case with Sjögren's syndrome combined with two other diseases, who obtained better motor and sensory recovery after 2-courses of laser therapy. A close relationship between motor/ sensory function and the dorsolateral prefrontal cortex was observed from the brain function images.

Keywords: Transverse Myelitis; Laser Irradiation; Laser Phototherapy; Dorsolateral Prefrontal Cortex; Single Photon Emission Computed Tomography; Berg Balance Score

Abbreviations: TM: Transverse Myelitis; GBS: Guillain-Barré syndrome; ECD: Ethyl Cysteinate Dimer; ILIB: Intravascular Laser Irradiation of Blood; SS: Sjögren's Syndrome; VAS: Visual Analogue Scale; SPECT: Single Photon Emission Computed Tomography; DLPFC: Dorsolateral Prefrontal Cortex

\section{Introduction}

Transverse myelitis (TM) is an autoimmune disease and often causes paralysis of the lower limbs, which motor impairment is characterized by flaccid paraplegia in the initial phase, and later a spastic paraparesis with tendinous hyperreflexia [1]. The Guillain-Barré syndrome (GBS), an acute inflammatory demyelinating polyradiculoneuropathy, is the most common cause of acute generalized paralysis, which clinical manifestations are sensorimotor disturbance and progressive limbs weakness. It usually presents as a paralysis that starts in the lower limbs and then progresses symmetrically upward. Deafferent pain syndrome is common in the early stages of recovery in GBS [2]. Combination of both TM and GBS will worsen the consequences, and results in various disabilities. Conventional treatments are effective in medical control, for instance, intravenous immunoglobulin [1], plasma exchange $[3,4]$. 
Motor and sensory recovery depend on adequate rehabilitation $[5,6]$, or virtual motor rehabilitation program [2]. However, those rehabilitative ways are slow and difficult in meeting some needs.

A novel method is always an expected way in order to have a faster recovery; therefore, the intravascular laser irradiation of blood (ILIB) comes with the tide of fashion. ILIB has been applied to multiple disciplines like cardiovascular events $[7,8]$, rheumatology-related arthritis [9-11], fibromyalgia [12], hepatology [13], obstetrics (parturient patients for postpartum mastitis and nipple soreness) [14] and diabetes mellitus [15-17], as its properties of increasing microcirculation and improving oxygen supply. It is reported with efficacy in facilitating the circulation and increased wound healing rate $[18,19]$. We herein report a case with TM combined with GBS, who was treated with ILIB and obtained better motor and sensory recovery.

\section{Case Presentation}

A 43-year-old woman with underlying Sjögren's syndrome (SS) and experienced a prior episode of acute gastroenteritis, which caused to development of TM and GBS 3-4 years ago. Bilateral lower limbs numbness and weakness (muscle power score 2-3) caused her as to disable, and under the standard treatment of steroid pulse therapy and immunomodulators, she could reluctantly walk with the help of quadricane. However, severe neuropathic pain at T4-T5 level with Visual Analogue Scale (VAS): 8-10 persisted without any recovery at all. She came to our outpatient department for better walking ability and pain relief. Berg Balance Score recorded as $8 / 56$. The regional cerebral perfusion scan with single photon emission computed tomography (SPECT) was performed and showed decreased activities in the frontal area. Two courses of ILIB were provided (ten consecutive days each course with resting interval of 7-14 days). The procedure of ILIB was mentioned as before [20]. In brief, insertion of an optic fiber was inserted into a superficial vein in order to convey laser light.

Helium-neon (He-Ne) laser (YJ-ILIB-5, Bio-ILIB Human Energy Ltd, Taiwan) was applied with wavelength of $632.8 \mathrm{~nm}$, power output 2.5 to $3.5 \mathrm{w} / \mathrm{cm}^{2}$, power intensity 1.79 to $2.04 \mathrm{w} / \mathrm{cm}^{2}$, energy 12.6 to $14.4 \mathrm{~J}$, energy density $6428.57 \mathrm{~J} / \mathrm{cm}^{2}$, and irradiation time $3600 \mathrm{~s} /$ session. We adjusted power output depending on the patient's responses. After completeness of two courses of ILIB, neuropathic pain diminished to VAS 1-2, and the weakness of the both lower limbs improved (muscle power from 2-3 to 4-5). We tested her posture performance, which showed independent standing without obvious tilting nor body shaking, standing with eyes closed independently, and standing on right leg 3.5 seconds and left leg 2.5 seconds, even still difficulty in jumping on one leg. The second Berg Balance Score recorded as 46/56. She could walk steadily for 3 meters, staggering while turning around. She could walk with minimal help but need a pause when she made a turn (Figure 1). The second brain SPECT showed increased activities in the frontal area (Figure 2).
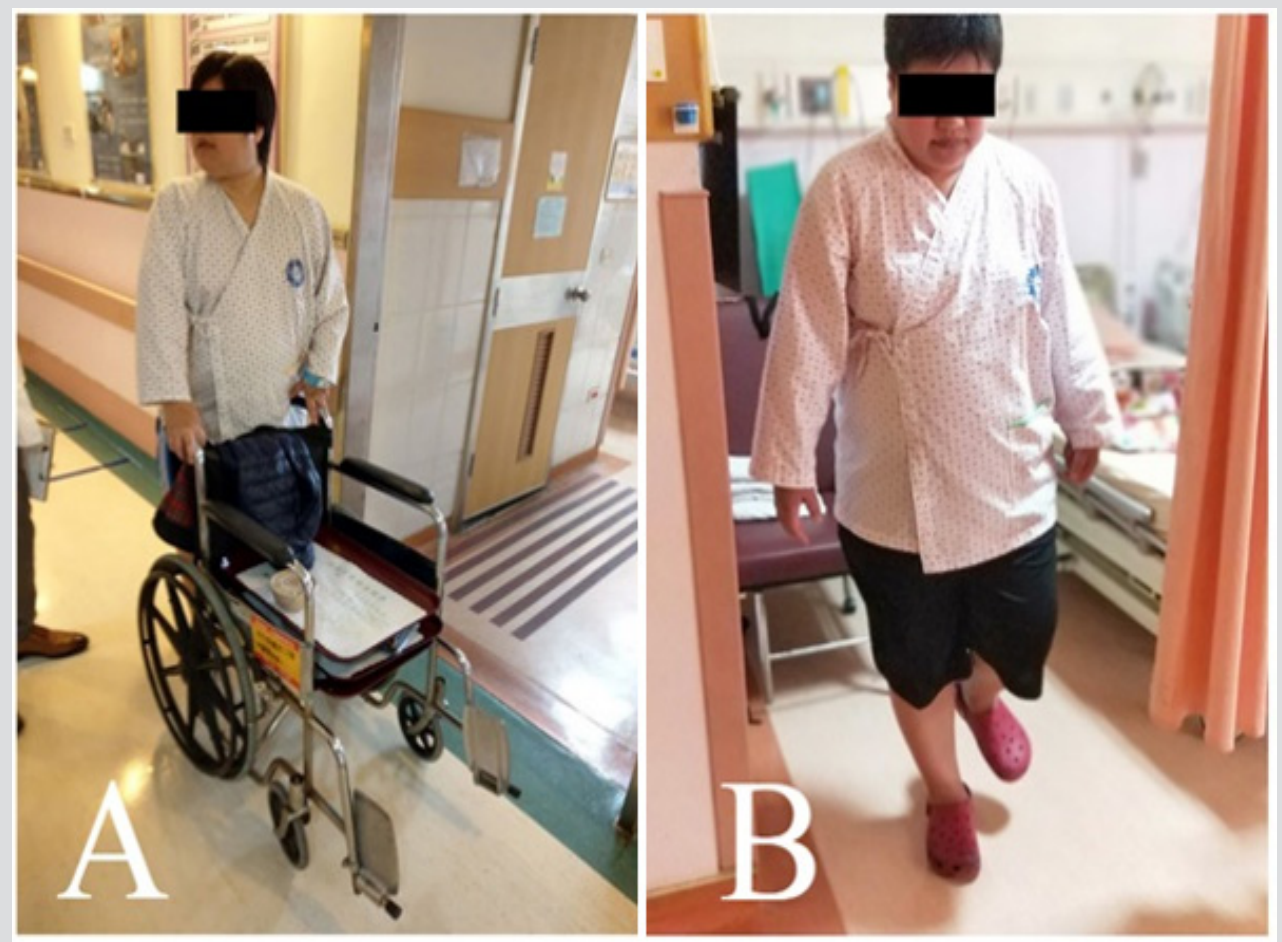

Figure 1: Walking ability of the patient. (A) She barely walked with the help of quadricane or wheelchair before the ILIB. (B) She could walk with minimal help but need a pause when she made a turn. 


\section{A. Before the ILIB intervention}

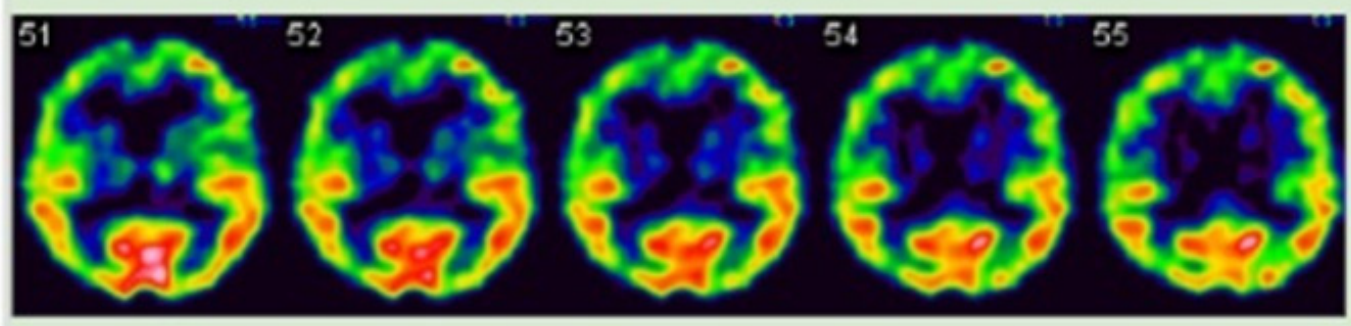

\section{B. After the ILIB intervention}

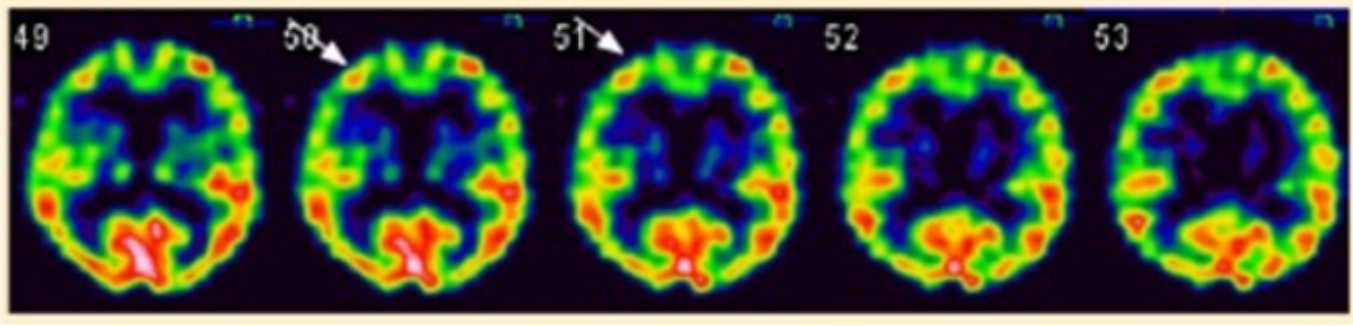

Figure 2: Tc-99m ethyl cysteinate dimer (ECD) brain perfusion SPECT study. Before (A) and after (B) the intervention of ILIB, there was increased uptake in the right frontal area, particularly in dorsolateral prefrontal cortex (arrow).

\section{Discussion}

Medical conditions of our case were complex because the causes of the patient's disability are multiple diseases, which were attributed by GBS, TM, and her underlying SS. Traditional medications and therapy help the patient overcome the acute stage. Even Sadowsky et al. [6] applied well-known neurophysiologic rules to post-TM rehabilitation in order to have reality impact on adaptive residual functional capabilities. Sadly, chronic sub-disability remained unresolved. We used brain SPECT to assess the brain function before and after the ILIB and found a strong relationship between pain and lower limbs function and frontal lobe activity. Motor cortex, which located in the dorsal precentral gyrus, consists of primary motor cortex, premotor cortex and supplementary motor area. Of motor cortex, the dorsolateral prefrontal cortex (DLPFC) plays an important role in motor planning, organization and regulation [21]. SS have been demonstrated to have high rate of alterations in cerebral blood flow, no matter what psychoneurological symptomatic and asymptomatic, which changes were mostly localized in the prefrontal and frontal areas, occipital lobes and occipitoparietal area, and less frequently so in the temporal, parietal and central areas [22]. Two-course ILIB reversed the deficit in DLPFC of our case. Not only motor deficit, but also pain perception is associated with DLPFC.

In the review article from Seminowicz \& Moayedi [21] stated that patients with chronic back pain, migraine, trigeminal neuropathic pain, hypnic headache, chronic post-traumatic headache, hip osteoarthritis and complex regional pain syndrome have reduced DLPFC gray matter, compared with healthy subjects. Our patient had neuropathic pain at the level of T4-T5, which pain had strong relationship with DLPFC, and the pain improved dramatically evidenced from increased uptake in the DLPFC in the second brain SPECT. The benefits obtained from ILIB were also shown from the posture-balance tests of our case. Balance improvement is assumed as subsequence to pain relief and motor function recovery. The decrease of blood flow of the region in the motor cortex leads to functional defect over lower limbs. ILIB is a therapeutic tool for facilitating circulation, including frontal area of the cortex especially in our case, which may improve the lower limbs function. One of our studies has shown that ILIB can improve perfusion of contralateral cerebellum during diaschisis after an ischemic stroke [20]. The ILIB also plays a role of immunomodulator as it directly or indirectly affects the cells of the immune system, which was unbalanced in our case with underlying SS. The post-treatment imaging study revealed that the ILIB was effective in facilitating circulation around the frontal area of the cortex, which improved the clinical performance especially pain and lower limbs function.

\section{Conclusion}

Both brain SPECT and posture/ balance tests provided us a direct evidence of positive correlation between ILIB intervention and clinical performance. As for the patient's reality concern and daily rehabilitation, the intervention of ILIB could offer a supplementary treatment for the symptom's relief and better life quality.

\section{References}

1. Sá MJ (2009) Acute transverse myelitis: a practical reappraisal. Autoimmun Rev 9(2): 128-131. 
2. Albiol Pérez S, Forcano García M, Muñoz Tomás MT, Manzano Fernández P, Solsona Hernández S, et al. (2015) A novel virtual motor rehabilitation system for Guillain-Barré syndrome. Two single case studies. Methods Inf Med 54(2): 127-134.

3. Chevret S, Hughes RA, Annane D (2017) Plasma exchange for GuillainBarré syndrome. Cochrane Database Syst Rev 2: CD001798.

4. Islam B, Islam Z, Rahman S, Endtz HP, Vos MC, et al. (2018) Small volume plasma exchange for Guillain-Barré syndrome in resource-limited settings: a phase II safety and feasibility study. BMJ Open 8(8): e022862.

5. Meythaler JM (1977) Rehabilitation of Guillain-Barré syndrome. Arch Phys Med Rehabil 78(8): 872-879.

6. Sadowsky CL, Becker D, Bosques G, Dean JM, McDonald JW, et al. (2011) Rehabilitation in transverse myelitis. Continuum (Minneap Minn) 17(4): 816-830.

7. Kipshidze NN, Chapidze GE, Bokhua MR, Marsagishvili LA, Dolidze NK, et al. (1990) Intravascular laser therapy of acute myocardial infarction. Angiology 41(9 Pt 2): 801-808.

8. Derkacz A, Protasiewicz M, Rola P, Podgorska K, Szymczyszyn A, et al. (2014) Effects of intravascular low-level laser therapy during coronary intervention on selected growth factors levels. Photomed Laser Surg 32(10): 582-587.

9. Timofeyev VT, Poryadin GV, Goloviznin MV (2001) Laser irradiation as a potential pathogenetic method for immunocorrection in rheumatoid arthritis. Pathophysiology 8(1): 35-40.

10. Chiran DA, Litscher G, Weber M, Ailioaie LM, Ailioaie C, et al. (2013) Intravenous laser blood irradiation increases efficacy of etanercept in selected subtypes of juvenile idiopathic arthritis: an innovative clinical research approach. Evid Based Complement Alternat Med 2013: 168134.

11. Chiran DA, Weber M, Ailioaie LM, Moraru E, Ailioaie C, et al. (2014) Intravenous laser blood irradiation and tocilizumab in a patient with juvenile arthritis. Case Rep Med 2014: 923496.

12. Momenzadeh S, Abbasi M, Ebadifar A, Aryani M, Bayrami J, et al. (2015) The intravenous laser blood irradiation in chronic pain and fibromyalgia. J Lasers Med Sci 6(1): 6-9.

\section{ISSN: 2574-1241}

DOI: 10.26717.BJSTR.2019.14.002572

Shin - Tsu Chang. Biomed J Sci \& Tech Res

(C) This work is licensed under Creative

Submission Link: https://biomedres.us/submit-manuscript.php
13. Babaev AV, Gogolev DE, Reiner OV, Korochkin IM, Fandeev AV, et al. (2012) Effect of intravenous low-intensity laser irradiation of the blood on clinical and laboratory parameters of hepatocellular insufficiency. Bull Exp Biol Med 153(5): 754-757.

14. Mokmeli S, Khazemikho N, Niromanesh S, Vatankhah Z (2009) The application of low-level laser therapy after cesarean section does not compromise bloodprolactin levels and lactation status. Photomed Laser Surg 27(3): 509-512.

15. Kazemi Khoo N, Iravani A, Arjmand M, Vahabi F, Lajevardi M, et al. (2013) A metabolomic study on the effect of intravascular laser blood irradiation on type 2 diabetic patients. Lasers Med Sci 28(6):1527-1532.

16. KazemiKhoo N, Ansari F (2015) Blue or red: which intravascular laser light has more effects in diabetic patients? Lasers Med Sci 30(1): 363366.

17. Kazemi Khoo N, Sarafnejad AF, Ansari F, Mehdipour P (2016) Modifying effect of intravenous laser therapy on the protein expression of arginase and epidermal growth factor receptor in type 2 diabetic patients. Lasers Med Sci 31(8): 1537-1545.

18. Huang SF, Tsai YA, Wu SB, Wei YH, Tsai PY, et al. (2012) Effects of intravascular laser irradiation of blood in mitochondria dysfunction and oxidative stress in adults with chronic spinal cord injury. Photomed Laser Surg 30(10): 579-586.

19. Mikhaylov VA (2015) The use of Intravenous Laser Blood Irradiation (ILBI) at 630-640 nm to prevent vascular diseases and to increase life expectancy. Laser Ther 24(1): 15-26.

20. Yang WH, Lin SP, Chang ST (2017) Case report: Rapid improvement of crossed cerebellar diaschisis after intravascular laser irradiation of blood in a case of stroke. Medicine (Baltimore) 96(2): e5646.

21. Seminowicz DA, Moayedi M (2017) The dorsolateral prefrontal cortex in acute and chronic pain. J Pain 18(9): 1027-1035.

22. Lass P, Krajka-Lauer J, Homziuk M, Iwaszkiewicz-Bilikiewicz B, Koseda M, et al. (2000) Cerebral blood flow in Sjögren's syndrome using 99TcmHMPAO brain SPET. Nucl Med Commun 21(1): 31-35.

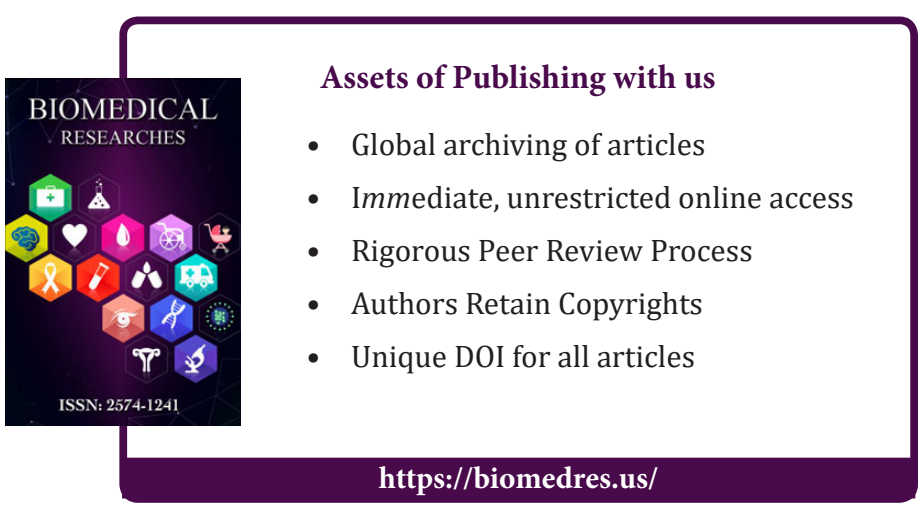

